\title{
Modelos econométricos de elección desde la economía del comportamiento: Modelamiento de elección discreta basada en costo emocional aleatorio - Aplicación a la industria agroquímica Colombiana.
}

Econometric models of choice from behavioral economics: Modeling of discrete choice based on random emotional cost - Application to the Colombian agrochemical industry. shorttitle

Gabriel Contreras ${ }^{\mathrm{a}}$

gabriel.contreras@sinnetic.com

\begin{abstract}
Resumen
Los modelos económicos ortodoxos, proponen que el ser humano es racional, egoísta y maximizador para hacer sus elecciones de consumo. Evidencia desde la economía del comportamiento reta estos supuestos planteando nuevos modelos para estudiar la elección humana. Estudiando el proceso de elección de productos de cuidado de cultivo en productores de tomate en Colombia, la presente investigación busco comparar estadística y conceptualmente los modelos RUM (Random Utility Maximization) y RRM (Random Regret Minimization) construidos vía modelamiento de elección discreta concluyendo que los modelos RRM logran mejor bondad de ajuste para describir el comportamiento de elección y compra de nematicidas en muestras de productores de tomate colombianos por lo que constituyen una alternativa viable para diseñar nuevos productos, estimar su participación potencial en el mercado y fijarles precio.
\end{abstract}

Palabras clave: Modelamiento de elección discreta, RUM (Random Utility Maximization), RRM (Random Regret Minimization), Economía del comportamiento, Comportamiento de elección..

\begin{abstract}
The orthodox economical models propose that the human being is rational, selfish and maximizing to make their consumption choices. The evidence from behavioral
\end{abstract}

${ }^{a} \mathrm{XXXXXXXXXXXXXXXXX}$ 
economics challenges these assumptions by proposing new models to study human choice. Studying the process of choosing crop care products in tomato growers in Colombia, this research sought to compare statistically and conceptually the RUM (Random Utility Maximization) and RRM (Random Regret Minimization) models built via modeling of discrete choice concluding that RRM models achieve better goodness of fit to describe choice behavior and purchases of nematicides in samples of Colombian tomato producers, so they constitute a viable model for designing new products, estimating their potential market share and fix prices using psychological and economical principles.

Keywords: Discrete choice models, RUM (Random Utility Maximization), RRM (Random Regret Minimization), Behavioral economics, Choice behavior..

\section{Introducción}

Desde que se encontraron puntos de dialogo entre la psicología y la microeconomía para describir, explicar, predecir e intervenir en la forma como los seres humanos distribuyen recursos limitados (tiempo y dinero) entre las diferentes alternativas del mercado y así comprender en profundidad el comportamiento de elección (Bekker-Grob, Ryan, Gerard, 2012) los experimentos de elección discreta, por sus siglas en ingles DCM (Discrete Choice Models), se han posicionado como una herramienta de primera mano para estudiar el comportamiento de elección en escenarios aplicados, posiblemente por su amplia sincronía con el proceso de elección en "la vida real" si se compara con las metodologías convencionales de análisis conjunto y escalas actitudinales (Mangham, Hanson, McPake, 2009).

Bajo DCM los productos, servicios o programas pueden ser descritos por sus características o atributos y la preferencia de las personas frente a estas propuestas, dependerán de los niveles de dichos atributos (Louviere, Hensher, Swait, 2000). La importancia relativa de los atributos, los sacrificios ocultos tras la elección que las personas hacen al escoger una alternativa y las predicciones probabilísticas de los resultados del proceso de elección que ejecutan las personas pueden ser evaluados y analizados en un conjunto de escenarios en donde los participantes experimentales pueden escoger entre una o más propuestas hipotéticas que son producto de la combinación de los atributos del producto (Hensher, Rose, Greene, 2005).

En el contexto de la fijación de precios a innovaciones agro-farmacéuticas, casi sin excepción, los investigadores han asumido un modelo basado en el supuesto que el agricultor compra los productos de cuidado de cultivo (Insecticidas, herbicidas y fungicidas) buscando maximizar el resultado de su elección (Louviere Lancsar, 2009). El proceso de elección basado en la maximización de la utilidad asume que los agricultores eligen los productos de cuidado de cultivo que le generen una mayor relación costo beneficio generando mayor utilidad; los modelos DCM basados en este supuesto se conocen como Estructuras de Maximización en la Utilidad Aleatoria o por sus siglas en ingles RUM (Random Utility Maximization) (McFadden D. , 1974); el concepto de aleatoriedad proviene del hecho que el investigador no tiene 
acceso directo a los procesos conductuales encubiertos "mente" del agricultor. La conducta pública durante el experimento de elección es conocido como componente simétrico u observado de la utilidad que se construye tras la combinación lineal entre los parámetros del modelo y los atributos de los productos de cuidado de cultivo que se presentan al agricultor de manera combinada formando escenarios de elección durante el experimento.

Aunque los modelos RUM se han convertido en un estándar para fijar el precio a nuevos productos de cuidado de cultivo al igual que para anticipar su participación en el mercado, asumir un modelo lineal aditivo que describa el proceso de decisión vía maximización de utilidad puede ser restrictivo para describir el comportamiento humano. Algunos avances relativamente recientes retan la idea de maximización de utilidad como supuesto subyacente a las elecciones humanas (Arentze Timmermans, 2007) pese a esto, los esfuerzos investigativos se han propuesto crear modelos de comportamiento de elección más realistas que han resultado ser menos parsimónicos ya que requieren más parámetros en comparación con los modelos RUM incrementando los grados de libertad en la estructura probabilística del modelo, tal es el caso de los modelos logit multinomiales (Zhang, Timmermans, Borgers, Wang, 2004). También se han sugerido modelos de concavos de superficie de respuesta que han demostrado alta eficiencia predictiva pero su implementación requiere sintaxis de código de alta complejidad quedando por ahora sujetos a los ambientes académicos y de ingeniería computacional haciendo difícil su aplicación en escenarios empresariales (Kivetz, Netzer, Srinivasan, 2004).

Desde el 2009 se comienzan a generar desarrollos de nuevos modelos conceptuales y metodológicos para estudiar comportamiento de elección principalmente en el sector del transporte basado en la noción de reducción de costos emocionales ocultos en el proceso de elección para analizar los datos de DCM (Chorus C. G., 2010) También conocidos como RRM por sus siglas en inglés (Random Regret Minimization). Esta alternativa de modelamiento mantiene altos niveles de trazabilidad y parsimonia al igual que el RUM sin embargo mantiene características deseables en el estudio del comportamiento de elección: a. Conductas cuasi compensatorias y b. efectos compromisorios. El presente estudio introduce la metodología RRM para modelar decisiones de cuidado de cultivo en productores de tomate y así investigar su usabilidad en contextos agro-farmacéuticos reales para fijación de precio a nuevos productos. La motivación al hacer este estudio se basa en resultados de estudios en comportamiento de elección cuyos resultados sugieren que la minimización de la decepción anticipada es un factor clave en elecciones que se consideran difíciles e importantes (Zeelenberg Pieters, 2007) Elegir un producto de cuidado de cultivo puede ser una decisión importante y difícil dadas las consecuencias de una equivocación que se traducen en pérdidas económicas y de productividad para el agricultor quien en el caso del tomate hace inversiones sostenidas por 4 meses promedio para esperar una productividad que le permitirá pagar deudas, subsistir y tener ingresos para una siguiente inversión. 


\section{Modelos de elección y formulación desde eco- nomía del comportamiento}

Los modelos RUM basan su fundamentación estadística en la hipótesis que el agricultor realiza sus elecciones buscando maximizar la utilidad. Desde su conceptualización matemática, el modelo asume que la utilidad latente de la alternativa $i$, $U_{i}$, puede ser descompuesta en dos partes aditivas: un componente simétrico explicativo, $V_{i}$, identificado como una función lineal generalizada de las características del producto, $\beta_{m} * X_{i m} ;$ y un segundo componente aleatorio o inexplicable, $\epsilon_{i}$. Este segundo es la porción estocástica de la función de utilidad la cual se asume idénticamente distribuida e independiente (ver ecuación 1).

$$
U_{i}=V_{i}+\epsilon_{i}=\sum \beta_{m} * X_{i m}+\epsilon_{i}
$$

Dada la distribución del término de error, y asumiendo que el consumidor elige las alternativas con máxima utilidad, las probabilidades de elección, $P_{i}$, están dadas por la siguiente formulación de tipo logit (ver ecuación 2).

$$
P(i)=\frac{e^{V_{i}}}{\sum_{i} e^{V_{i}}}
$$

Por su parte el enfoque RRM se basa en minimizar el arrepentimiento sobre la elección más que maximizar la utilidad cuando el agricultor elige entre dos o más alternativas de cuidado agro-farmaceuta de cultivo. Evidencia sobre la minimización del arrepentimiento ha sido expuesta en diferentes estudios teóricos y empíricos desde hace dos décadas (Bell, 1982) y con novedosos mecanismos investigativos se ha sido validado recientemente (Muller, Kroll, \& Vogt, 2012).

Los modelos RRM suponen que dado que un agro-fármaco está compuesto por varios atributos, ejemplo: amplio espectro, días control, seguridad, toxicidad etc., y cada atributo tiene niveles, tomando como ejemplo los días control: control del insecto por 5 días, control por 10 días, control por 20 días etc., es muy difícil que todos los atributos del producto logren maximizar la utilidad desde la elección del agricultor, por lo que este debe aceptar que algunos de los atributos del producto tengan niveles no tan óptimos siempre que la totalidad de los atributos generen una experiencia de optimización; desde esta perspectiva el arrepentimiento del agricultor se presenta cuando una alternativa no elegida supera en por lo menos un atributo importante a la alternativa elegida (Chorus., 2012). La estructuración matemática del arrepentimiento por parte del agricultor puede darse como muestra la ecuación 3.

$$
R R_{i}=R_{i}+\epsilon_{i}=\sum_{j \neq i} \sum_{m} \ln \left(1+\exp \left[\beta_{m} *\left(x_{j m}-x_{i m}\right)\right]+\epsilon_{i}\right.
$$

En esta concepción, el arrepentimiento concentrado ante una alternativa $i, R R_{i}$, 
cuenta con dos porciones, una explicativa formada por la adición de efectos de los atributos del producto no elegido $x_{j} m$ en comparación con el producto elegido $x_{i} m$ más un componente estocástico de error $\epsilon_{i}$ el cual tiene distribución idéntica e independiente con valores extremos tipo I. Dada esta distribución del componente aleatorio y asumiendo que el consumidor elige la alternativa que le genere menos arrepentimiento, la probabilidad de elección vendrá dada por la expresión logit expuesta en la ecuación 4.

$$
P(i)=\frac{\exp \left(-R_{i}\right)}{\sum_{i=1 \ldots J} \exp \left(-R_{i}\right)}
$$

El término $\ln \left(1+\exp \left[\beta_{m} *\left(x_{j m}-x_{i m}\right)\right]\right.$ mide la cantidad de arrepentimiento que emerge al comparar la alternativa $i$ contra la alternativa $j$ en términos de un atributo particular denominado $x_{m}$; esta formulación implica que el arrepentimiento tiende a cero cuando la alternativa no elegida $\mathrm{j}$ presenta un desempeño peor comparado con el desempeño ofrecido por la alternativa $i$; cuando el caso contrario ocurre y la alternativa elegida presenta un peor desempeño en el atributo en comparación con la no elegida, el arrepentimiento tiende a 1. El parámetro $\beta_{m}$ es una aproximación de la forma de la función de arrepentimiento para el atributo $x_{m}$.

Desde la economía del comportamiento, los dos modelos difieren entre sí desde dos perspectivas que son: a. Comportamiento semi-compensatorio y b. Efecto comprometedor (Chorus C. G., 2010), RUM se muestra como una alternativa de comportamiento completamente compensatorio mientras que RRM se muestra semicompensatorio, un comportamiento completamente compensatorio ocurre cuando el bajo desempeño del producto en un atributo se compensa por completo por el buen desempeño del producto en otros, por otro lado RRM asume que un mejor desempeño de un atributo de producto que ya se desempeña mejor que la alternativa no elegida tiene un bajo efecto llevando el arrepentimiento a cero, mientras que un mal desempeño de un atributo comparado con la alternativa no elegida acelera el arrepentimiento a 1, como resultado este modelo asume que el bajo desempeño de un atributo puede ser compensado por un alto desempeño en otro atributo siempre que este segundo sea igualmente importante que el primero.

El efecto comprometedor emerge por limitaciones cognoscitivas del consumidor, la cantidad de beneficios de alto desempeño que un producto promete son extensos en comparación con los atributos que pueden presentar mal desempeño (Simonson, 1992). Dado que la memoria es un recurso limitado, el heurístico de almacenamiento en el consumidor sugiere que es más económico almacenar los atributos de bajo desempeño dado que ocupan menos espacio nemónico por lo tanto RUM exige que el consumidor comprometa más recursos de memoria en comparación con RRM.

El presente estudio busca analizar el desempeño psicométrico de tres alternativas de modelamiento de comportamiento de elección ante una innovación agrofarmacéutica orientada a controlar nematodos en el cultivo de tomate. Las tres alternativas son modelo RUM, modelo RRM y modelo hibrido, los tres son modelos lineales generalizados. 
El estudio tuvo tres fases: a. fase 1: Estudio cualitativo con el objetivo de explorar en productores de tomate cuales serían los factores claves de compra de un nematicida profundizando psicológicamente en sus necesidades redactando un listado de posibles elementos persuasivos por los cuales un agricultor compraría el producto. B. fase 2: Estudio cuantitativo con el objetivo de reducir la lista de factores claves de compra encontrados en la fase 1 y así tener mayores elementos de juicio para elaborar escenarios experimentales de la fase 3. C. fase 3: Estudio cuantitativo de exposición al agricultor a escenarios experimentalmente diseñados bajo combinación ortogonal niveles de los atributos encontrados en la fase 1 y filtrados en la fase 2 .

\section{FASE I}

\subsection{Participantes}

Partiendo de información proveniente de la encuesta nacional de agricultura del año 2011 (DANE, 2011) se seleccionaron 6 departamentos por sus niveles de producción de tomate a saber: Antioquia, Boyacá, Cundinamarca, Huila, Risaralda y Santander. En cada uno de estos departamentos, se seleccionó a conveniencia, muestreo no probabilístico, 7 agricultores que cumplieran con las siguientes características: a. Más de 2 años de experiencia en el cultivo de tomate, b. Menos de 4 hectáreas de tierra sembradas por temporada, c. Dueños de las tierras, d. Principales tomadores de decisiones sobre los productos a comprar y aplicar para el cuidado de su cultivo, e. No haber participado en estudios por encuesta asociados a productos para cuidado de cultivo en los seis meses anteriores al ejercicio. Esto generó una muestra de 42 agricultores con edades comprendidas entre los 35 y 57 años $(\mu=41 ; \sigma=3,2)$.

\subsection{Instrumento}

Se construyó una guía de entrevista semi estructurada siguiendo los principios metodológicos de la entrevista en profundidad que según Martin y Gaskel (2000) citados por Velandia y López es un recurso de investigación cualitativa y mixta que "Se puede definir como una interacción entre dos personas, en la cual el investigador formula determinadas preguntas relativas al tema en investigación, mientras que el entrevistador proporciona verbalmente la información que le es solicitada. Es un intercambio de ideas, significados, sentimientos sobre el mundo y los eventos, cuyo principal medio son las palabras". (Velandia López, 2008). La entrevista fue conducida por psicólogos y antropólogos graduados y previamente entrenados en las realidades sociales, procedimentales y técnicas del cultivo de tomate y los agricultores.

Se construyó una guía de entrevista semi estructurada siguiendo los principios metodológicos de la entrevista en profundidad que según Martin y Gaskel (2000) 
citados por Velandia y López es un recurso de investigación cualitativa y mixta que "Se puede definir como una interacción entre dos personas, en la cual el investigador formula determinadas preguntas relativas al tema en investigación, mientras que el entrevistador proporciona verbalmente la información que le es solicitada. Es un intercambio de ideas, significados, sentimientos sobre el mundo y los eventos, cuyo principal medio son las palabras". (Velandia López, 2008). La entrevista fue conducida por psicólogos y antropólogos graduados y previamente entrenados en las realidades sociales, procedimentales y técnicas del cultivo de tomate y los agricultores.

Partiendo de lo general a lo particular, se exploraron las necesidades de los agricultores alrededor de los nematodos como problema fitosanitario y sus prácticas de cultivo haciendo especial énfasis en aquellos espacios que los productos actuales propuestos en el mercado han descuidado ya sea por omisión o por propuestas de producto técnicamente débiles. Análisis de información.

Desde el método de teoría fundamentada (Howard-Payne, 2016), los audios de las entrevistas se procesaron mediante CAQDAS (Computer-Assisted Qualitative Data Analysis Software) Nvivo 10. De forma procedimental se codificó y clasificó la información con el objetivo de cuantificar categorías de análisis pre-existentes y emergentes (Franzosi, Doyle, McCelland, Putnam Rankin, Vicari, 2013)

\subsection{Resultados}

Tras el análisis de la información se logró aislar 20 factores clave que motivarían al agricultor a comprar un nematicida, estos se agruparon en 6 familias conforme se evidencia en la tabla 1.

\section{FASE II}

\subsection{Participantes}

Retomando la misma distribución geográfica de la fase 1, en un segundo momento de muestreo, con agricultores diferentes a los de la fase anterior, se seleccionó a conveniencia, muestreo no probabilistico, 20 agricultores por departamento conformando una muestra por conveniencia de 120 agricultores bajo el mismo perfil previamente descrito con edades comprendidas entre los 38 y 57 años de edad ( $\mu=44 ; \sigma=3,2)$. Instrumento. Se generaron escenarios de elección bajo la metodología de escalamiento de máxima diferencia significativa MaxDiff (Anderson Wiley, 1992) en donde los factores claves de compra descritos en la tabla 1 se combinaron ortogonalmente en grupos de 4 conformando 32 grupos. En cada grupo, luego de una lectura de cada elemento se le preguntaba al agricultor cuál de los factores clave de compra era el más importante, de igual forma se cuestionó por el menos importante. Resultados. El análisis de resultados permitió reducir los 20 factores de compra a 7 conforme la tabla 2. El criterio de elección se basó en 
Tabla 1: Necesidades más importantes a la hora de comprar insecticidas. Aislamiento cualitativo y agrupación en familias.

\begin{tabular}{|c|c|c|}
\hline Familia & Necesidad & Descripción. \\
\hline \multirow{4}{*}{$\begin{array}{l}\text { Amigable con } \\
\text { el ecosistema }\end{array}$} & Residualidad. & $\begin{array}{l}\text { Que el Nematicida debe muy bajo residuo químico } \\
\text { en el producto final. }\end{array}$ \\
\hline & Toxicidad. & $\begin{array}{l}\text { Que el Nematicida no sea dañino para la salud } \\
\text { del agricultor. }\end{array}$ \\
\hline & Eco amigable. & $\begin{array}{l}\text { Que el Nematicida no contamine aguas y } \\
\text { el ambiente en general. }\end{array}$ \\
\hline & Fauno benéfico. & $\begin{array}{l}\text { Que el Nematicida sea específico y no afecte } \\
\text { insectos que son benéficos para el control de } \\
\text { hongos en el cultivo. }\end{array}$ \\
\hline \multirow{4}{*}{ Efectividad. } & Días control. & $\begin{array}{l}\text { Que el Nematicida se mantenga actuando } \\
\text { varios días después de ser aplicado. }\end{array}$ \\
\hline & Contundencia. & $\begin{array}{l}\text { Que el tiempo que demora el Nematicida en } \\
\text { controlar nematodos sea corto. }\end{array}$ \\
\hline & Resistencia al clima & $\begin{array}{c}\text { Que se peque en las raices y no se lave con } \\
\text { la lluvia. }\end{array}$ \\
\hline & Convivencia en mezcla. & $\begin{array}{l}\text { Que el Nematicida pueda mezclarse con otros } \\
\text { productos en tanque sin que pierda efecto. }\end{array}$ \\
\hline \multirow[b]{3}{*}{ Autorización. } & Regulación. & $\begin{array}{l}\text { Que el Nematicida este aprobado para venta y } \\
\text { uso en Colombia. }\end{array}$ \\
\hline & Modo de aplicación. & $\begin{array}{l}\text { Que se pueda aplicar tanto de manera foliar } \\
\text { como en suelo para obtener mejores efectos. }\end{array}$ \\
\hline & Resistencia. & $\begin{array}{c}\text { Que el Nematicida tenga una estructura química } \\
\text { que haga difícil que los insectos desarrollen } \\
\text { resistencia. }\end{array}$ \\
\hline \multirow{3}{*}{ Productividad. } & Vigor. & $\begin{array}{c}\text { Un Nematicida que haga que la planta genere } \\
\text { nuevas raices saludables. }\end{array}$ \\
\hline & Productividad. & $\begin{array}{l}\text { Un Nematicida que haga que la planta produzca } \\
\text { tomates más grandes y sanos. }\end{array}$ \\
\hline & & $\begin{array}{l}\text { Un Nematicida que no esterilice la tierra para } \\
\text { sembrar relativamente rápido después de cosechar. }\end{array}$ \\
\hline \multirow{3}{*}{$\begin{array}{l}\text { Distribución } \\
\text { y precio }\end{array}$} & Distribución. & $\begin{array}{l}\text { Un Nematicida que se pueda encontrar fácil en } \\
\text { épocas de cultivo. }\end{array}$ \\
\hline & Precio & Un Nematicida con precio razonable y justo. \\
\hline & Costo beneficio. & $\begin{array}{l}\text { Un Nematicida que cumpla lo que promete } \\
\text { peso a peso }\end{array}$ \\
\hline \multirow{2}{*}{$\begin{array}{l}\text { Recomendación } \\
\text { y reputación }\end{array}$} & $\begin{array}{l}\text { Reconocimiento. } \\
\text { Recomendación. }\end{array}$ & $\begin{array}{c}\text { Un Nematicida reconocido por los agricultores. } \\
\text { Un Nematicida recomendado por las personas } \\
\text { que venden agroquímicos. }\end{array}$ \\
\hline & & $\begin{array}{c}\text { Un Nematicida producido por una empresa } \\
\text { seria y reconocida. }\end{array}$ \\
\hline
\end{tabular}

Comunicaciones en Estadística, noviembre 2020, Vol. 13, No. 2 
el score logit estimado en los modelos Maxdiff aislando todos los coeficientes con signo positivo (Corrigan, Depositario, Nayga Jr, Wu, Laude, 2009)

Tabla 2: Estimación LOGIT del modelo MaxDiff seleccionando los factores más importantes para el agricultor a la hora de comprar insecticidas.

\begin{tabular}{|l|l|l|}
\hline Familia & Necesidad & MaxDiff Score. \\
\hline Amigable con el ecosistema & Residualidad. & 1,34 \\
\hline \multirow{2}{|}{ Efectividad. } & Días control. & 2,43 \\
\hline & Contundencia. & 3,44 \\
\cline { 2 - 3 } & Resistencia al clima & 2,33 \\
\hline Productividad. & Convivencia en mezcla. & 4,33 \\
\hline & Vigor. & 3,22 \\
\hline
\end{tabular}

\section{FASE III}

\subsection{Participantes}

Bajo la cobertura geográfica, método de selección y criterios de inclusión descritos para la fase I y II se seleccionó para cada geografía un número de agricultores proporcional a la producción reportada para cada geografía según DANE configurando una muestra de 300 participantes distribuidos conforme la tabla 3 . El $18 \%$ de ellos habían participado en fase 1 o 2 . Los participantes reportaron edades comprendidas entre los 37 y 57 años . $\left(\mu=42 ; \sigma^{2}=3,1\right)$

Tabla 3: Distribución muestral de la fase 3

\begin{tabular}{|l|l|}
\hline Departamento & Entrevistas realizadas \\
\hline Antioquia & 65 \\
\hline Boyacá & 60 \\
\hline Cundinamarca & 55 \\
\hline Huila & 55 \\
\hline Risaralda. & 35 \\
\hline Santander & 30 \\
\hline Muestra: & 300 \\
\hline
\end{tabular}

Comunicaciones en Estadística, noviembre 2020, Vol. 13, No. 2 


\section{Instrumento}

El listado de factores clave de compra aislados en fase II fue definido operacionalmente con ayuda de 4 ingenieros agrónomos con el objetivo de estructurar niveles de alto, medio y bajo desempeño para cada uno de los motivadores configurando la tabla de atributos y niveles descritos en la tabla 4. Se adicionó el factor precio el cual permite identificar líneas de restricción presupuestaria como factor clave de elección.

Tabla 4: Atributos y categorías tenidas en cuenta para el diseño del experimento DCM.

\begin{tabular}{|c|c|c|c|c|}
\hline Familia & Necesidad & Expresión de bajo desempeño. & Expresión de desempeño medio. & Expresión de alto desempeño \\
\hline Amigable con el ecosistema & Residualidad. & $\begin{array}{l}\text { El tomate podría contener } \\
\text { residuos del nematicida. }\end{array}$ & & $\begin{array}{l}\text { El tomate no tendría residuos } \\
\text { del nematicida. }\end{array}$ \\
\hline \multirow{4}{*}{ Efectividad. } & Días control. & $\begin{array}{l}\text { El producto controla nematodos } \\
\text { durante } 5 \text { días después de aplicado. }\end{array}$ & $\begin{array}{l}\text { El producto controla nematodos } \\
\text { durante } 9 \text { días después de aplicado. }\end{array}$ & $\begin{array}{l}\text { El producto controla nematodos durante } \\
\text { 12dias después de aplicado. }\end{array}$ \\
\hline & Contundencia. & $\begin{array}{l}\text { El control de nematodos se observa } \\
\text { después de la segunda aplicación. }\end{array}$ & & $\begin{array}{l}\text { El control de nematodos se observa } \\
\text { después de la primera aplicación. }\end{array}$ \\
\hline & Resistencia al clima & $\begin{array}{l}\text { El producto puede } \\
\text { lavarse con la lluvia. }\end{array}$ & & $\begin{array}{l}\text { El producto se adhiere a las raíces y } \\
\text { las fuertes lluvias no logran lavarlo. }\end{array}$ \\
\hline & Convivencia en mezcla. & $\begin{array}{l}\text { El nematicida no se puede aplicar } \\
\text { en mezcla con otros productos. }\end{array}$ & & $\begin{array}{l}\text { El nematicida puede aplicarse en mezcla } \\
\text { con fertilizantes de aplicación a tierra. }\end{array}$ \\
\hline \multirow[b]{2}{*}{ Productividad. } & Vigor. & $\begin{array}{l}\text { El producto no favorece } \\
\text { el desarrollo de nuevas raíces. }\end{array}$ & & $\begin{array}{l}\text { El producto favorece el } \\
\text { desarrollo de nuevas raíces. }\end{array}$ \\
\hline & Productividad. & $\begin{array}{l}\text { El producto no acelera } \\
\text { la productividad de la planta. }\end{array}$ & & $\begin{array}{l}\text { El producto incrementa en un } 5 \% \\
\text { los niveles de productividad de la planta. }\end{array}$ \\
\hline Precio & \multicolumn{4}{|c|}{8 niveles de variación entre COP8.000hastaCOP 30.000 dosis por hectárea. } \\
\hline
\end{tabular}

Los niveles de cada atributo del producto se combinaron ortogonalmente formando 20 escenarios compuestos por 4 productos hipotéticos más la opción de ninguno. Tras la cuidadosa lectura de cada escenario, el agricultor debía elegir una de las 5 alternativas como aquella que estaría dispuesto a comprar (Variable dependiente binaria), adicional a esto, para la alternativa seleccionada debía reportar la cantidad de tierra en hectáreas a la cual aplicaría el producto para probarlo.

\section{Análisis de resultados}

Los datos capturados fueron procesados haciendo uso de SAS STAT y SAS IML bajo Enterprise Guide 7,1. Se acudió a la biblioteca de modelos lineales generalizados mixtos (McGilchrist, , 1994) con estimación bayesiana de parámetros (Heydari , Miranda-Moreno, Liping, , 2014).

\subsection{Análisis de información}

Se programaron tres versiones del modelo DCM: a. Versión RUM, b. Versión RRM y c. Versión hibrida RUM-RRM; este último, es una expresión lineal generalizada 
que adiciona el componente de arrepentimiento como factor multiplicativo del impacto de los atributos de producto sobre la probabilidad de elección del mismo tal y como se observa en la ecuación 5. Los resultados comparativos de los tres modelos pueden evidenciarse en la tabla 5

$$
P(i)=\frac{\exp \left(V_{i} *-R_{j}\right)}{\sum_{j=1 \ldots J} \exp \left(V_{i} *-R_{j}\right)}
$$

Tabla 5: Estimación de modelos RUM, RRM e híbridos comparados.

\begin{tabular}{|c|c|c|c|c|c|c|c|}
\hline & \multicolumn{2}{|l|}{ RUM } & \multicolumn{2}{|l|}{ RRM } & \multicolumn{3}{|c|}{ Híbrido RUM-RRM } \\
\hline & Coef & $\mathrm{T}$ & Coef & $\mathrm{T}$ & coef & $\mathrm{t}$ & Tipo \\
\hline \multicolumn{8}{|l|}{ Parámetros de efecto fijo } \\
\hline Residualidad. & 0,545 & $3,01^{*}$ & 0,595 & $3,14^{*}$ & 0,595 & $3,16^{*}$ & RRM \\
\hline Días control. & 0,544 & $2,17^{* *}$ & 0,554 & $2,36^{* *}$ & 0,554 & $2,38^{*}$ & RRM \\
\hline Contundencia. & 0,511 & $4,12^{*}$ & 0,581 & $4,35^{*}$ & 0,581 & $4,36^{*}$ & RRM \\
\hline Resistencia al clima & 0,432 & $8,13^{* *}$ & 0,492 & $8,08^{* *}$ & 0,492 & $8,08^{*}$ & RRM \\
\hline Convivencia en mezcla. & 0,542 & $9,12^{*}$ & 0,542 & $10,36^{*}$ & 0,542 & $10,3^{*}$ & RRM \\
\hline Vigor. & 0,863 & $3,12^{*}$ & 0,903 & $3,61^{*}$ & 0,903 & $3,62^{*}$ & RRM \\
\hline Productividad. & 0,294 & $8,56^{*}$ & 0,374 & $8,94^{*}$ & 0,384 & $8,97^{*}$ & RRM \\
\hline Precio & $-0,543$ & $8,45^{*}$ & $-0,473$ & $8,44^{*}$ & $-0,443$ & $8,47^{*}$ & RRM \\
\hline \multicolumn{8}{|l|}{ Parámetros de efecto aleatorio } \\
\hline Constante (media (desviación)) & $3,66(4,21)$ & $5,64(6,8)$ & $2 ., 33(2,8)$ & $4,3(4,5)$ & $2,2(2,6)$ & $4,5(6,1)$ & \\
\hline Clasificación correcta & 0,75 & & 0,86 & & 0,80 & & \\
\hline Fasos positivos & 0,125 & & 0,07 & & 0,10 & & \\
\hline Log-verosimilitud & $-1,309$ & & $-1,182$ & & $-1,312$ & & \\
\hline $\mathrm{AIC}$ & 2,634 & & 2,312 & & 2,651 & & \\
\hline $\mathrm{BIC}$ & 2,684 & & 2,328 & & 2,689 & & \\
\hline $\mathrm{N}$ & 300 & & 300 & & 300 & & \\
\hline
\end{tabular}

- * Significancia al 95

- ** Significancia al 99

- $\mathrm{AIC}=$ Criterio de información de Akaike.

- $\mathrm{BIC}=$ Criterio de información bayesiano.

Desde el punto de vista estadístico, el modelo hibrido y el RRM tienen mejor capacidad predictiva del proceso de decisión de los productores de tomate ante la compra de productos orientados a control de nematodos presentando evidencia en favor de pensar que el agricultor no hace elecciones buscando maximizar la utilidad de las mismas sino evitando sentir fracaso o decepción ante un mal resultado. Esto se hace evidente no solo al ser los modelos con menor AIC y BIC, tambien muestran los mayores índices de clasificación correcta RNN: 86\%, híbrido: \%: 80\%.

Dentro del modelo RRM El atributo más importante para los productores de tomate es la promesa de vigor, hasta el punto que un nematicida que prometa que su aplicación incrementa la capacidad de la raíz de crecer y renovarse permitiendo entonces a la planta absorber mas nutrientes de la tierra tiene 0,9 más posibilidades de ser comprado que otro tipo de nematicida $(P<0,05)$. En segundo lugar la promesa de contundencia $(\beta: 0,5 P<0,05)$ permite ver que el agricultor se siente motivado a comprar un producto que rápidamente controle los síntomas y la expansión del problema en el cultivo. 
El perfil de nematicida con mayor elección por parte del agricultor por ser capaz de controlar los costos emocionales ocultos de acuerdo al modelo RRM se describe así: a. baja residualidad, b. 9 días control, c. Control después de la primera aplicación, d. Versatilidad en mezcla, e. alta adherencia a la hoja para que no se lave con el clima, f. favorece el desarrollo de nuevas raíces, g. Ayuda a incrementar la productividad de la planta hasta un $5 \%$. Para ilustrar el proceso de elección en función del precio, con la ecuación RRM presentada en la tabla 5, se construyó la curva de demanda que se puede observar en la gráfica 1. La curva de demanda expresa en $\mathrm{Y}$ el porcentaje de agricultores que eligen el producto en función del precio que se expresa en X. En la medida en la que se sube el precio, la demanda ante el perfil del producto más elegido por RRM tiende a decrecer, económicamente a esto se le conoce como elasticidad. El hecho que el producto se ponga caro y el agricultor deje de elegirlo, no quiere decir que se haya extinguido la necesidad, por lo cual emergen dos comportamientos sustitutos, a. Elección sobre otros perfiles de producto (competidores), b. Preferencia por no hacer aplicaciones.

Figura 1: Gráfica 1: Estimación de la demanda a diferentes escenarios de precio usando el modelo RRM seleccionado.

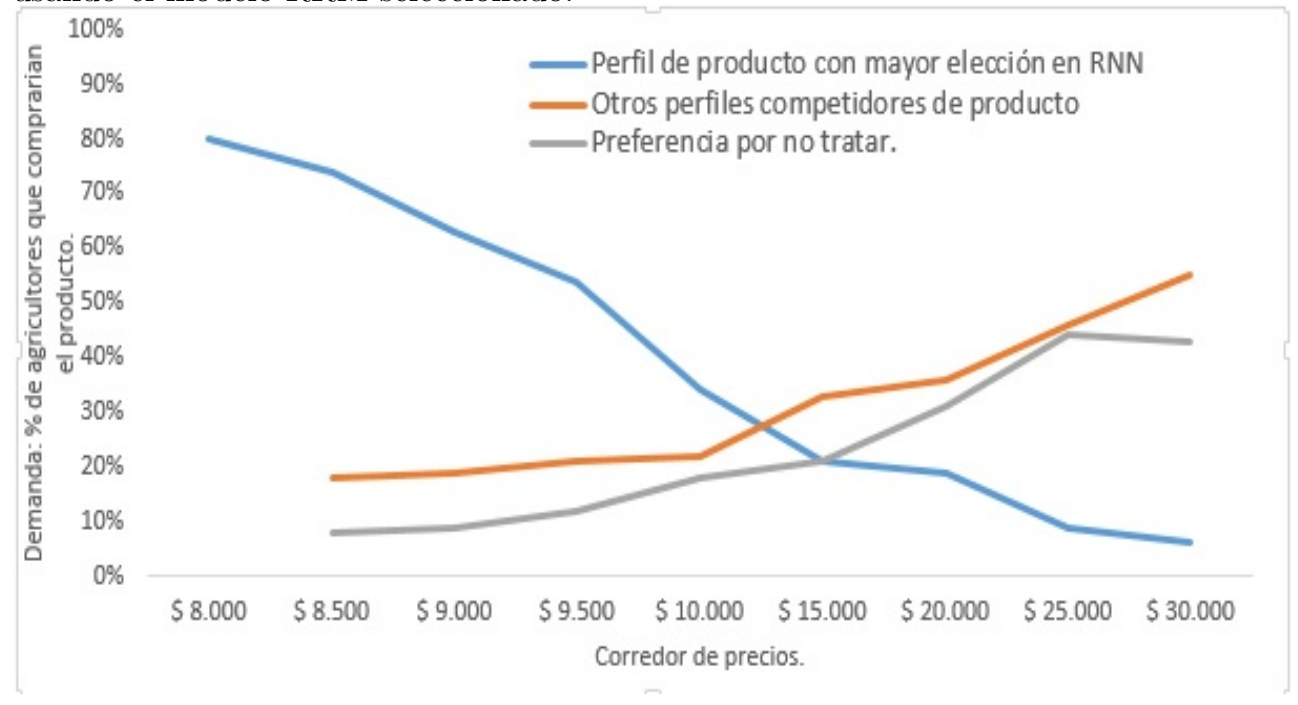

El lector debe notar como a COP12.000 el agricultor prefiere cualquier otro competidor en lugar del perfil de producto más elegido ya que este último es muy costoso; a COP 15.000 el agricultor prefiere simplemente no tratar o controlar nematodos porque percibe que la alternativa de tratamiento no justifica sus resultados, a estos dos puntos se les conoce como indiferencias ya que la demanda se ve afectada significativamente por comportamientos sustitutos (Sussex, Towse, Devlin, 2013.) Mediante un algoritmo SIMPLEX de optimización (Karimi Siarry, 2012) que busque crecer lo máximo en X (cobrar lo máximo posible por el perfil de producto), obteniendo lo máximo en Y (obteniendo la mayor cantidad de demanda posible) y alejándose lo máximo posible de la primera indiferencia de COP 12.000 se logra 
estimar un precio óptimo el cual se configura en COP 9.450 por hectárea para este nuevo producto, a este precio, se estima que el producto tendría una demanda cercana al 51Discusión. El modelo económico ortodoxo, define el ser humano como REM (Racional, Egoísta y Maximizador) y sobre esta visual formula modelos como el RUM ampliamente usado para definir el comportamiento del consumidor, estimar la demanda de nuevos productos y fijarles precio (Richardson McKie, 2005), sin embargo, evidencia se ha venido sumando para debatir estos tres principios como una máxima del comportamiento de elección (Kahneman, 2011). El presente documento presenta evidencia en favor del modelo RRM como alternativa explicativa del comportamiento de elección en agricultores y mecanismo metodológico para diseñar productos de cuidado de cultivo, fijar precio a estos diseños y estimar la demanda potencial de los mismos en el mercado. Esta alternativa supera el modelo convencional RUM en capacidad predictiva frente el comportamiento de elección del agricultor, tal como se muestra en la tabla 5 en los indicadores de clasificación correcta, BIC y AIC. El modelo RUM, centra su atención en la maximización de la utilidad que nuevos atributos del producto pueden representarle al agricultor (Nowlis Simonson, 1996), el modelo RRM se concentra en estudiar el sacrificio que debe hacer el agricultor para escoger cualquier opción del mercado (Chorus C. , 2012). La predicción del modelo microeconómico tradicional es que si el agricultor pudiera comprar el "mejor"producto, lo haría; el asunto es que no se sabe cual es, tendría que probarlos todos y tiene restricción de tiempo y presupuesto para lograrlo. Lo anterior implica que ante cualquier elección harbá una insertidumbre y una probabilidad de descepción (Chorus C. G., 2010). El modelo RRM presenta una expresión matemática para cuantificar el grado de descepción ante una elección; en economía del comportamiento, la opción de producto que mejor minimice este componente será la preferida por el agricultor tal y como se revela en la tabla 5 con la baja tasa de falsos positivos del modelo RRM vs los modelos RUM e híbrido (Chorus C. G., 2010). Esta propuesta de minimización de costos emocionales ocultos es diametralmente opuesta al modelo RUM y abre un nuevo paradigma para la fijación de precios de innovaciones agro-farmacéuticas (Cranenburgh, Angelo Guevara, Chorus, 2015). Independientemente del enfoque analítico, ya sea vía RUM, RRM o hibrido, el modelamiento de elección discreta es una herramienta de primera mano para la construcción de modelos de negocio que impliquen a. diseño de producto, b. estimación de participación potencial de mercado del producto diseñado y c. fijación de precio al nuevo producto teniendo en cuenta el comportamiento del consumidor y sus patrones de elección (Chorus C. , 2012)

\section{Referencias}

Carter R. (2014). Will I Hit My Year-End Numbers?. A Brand-Performance Forecasting Model. The Case for Benchmarking Sales and Advertising Spending. Jour-

nal of Advertising Research, 54(4), 398- 406. 
Brexendorf, T., Bayus, B., Keller, K. (2015). Understanding the Interplay Between Brand and Innovation Management: Findings and Future Research Directions. Journal of The Academic Marketing Science, 43, 548-557.

Aaker, D. (1996). Measuring brand equity across products and markets. California Management $\quad$ Review, $\quad 38(3), \quad 102-120$.

Gatignon, H., Xuereb, J.-M. (1997). Strategic Orientation Of The Firm And New Product Performance. Journal of Marketing Research, 34(1), 77.90.

Hoeffler, S., Keller, K. (2003). The Marketing Advantages Of Strong Brands. Journal of Brand Management, 10(10), 421-445.

Keller, K., Lehmann, D. (2006). Brand and Branding: Research Findings and Fu-

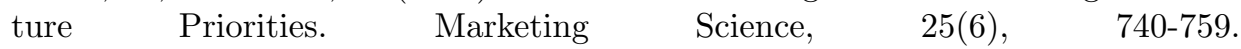

Liao, S., Cheng, C. (2014). Brand Equity and the Exacerbating Factors of Product Innovation Failures Evaluations: a Communication Effect Perspective. Journal of $\begin{array}{llll}\text { Business } & \text { Research, } & 67(1), & 2919-\end{array}$

Prins, R., Verhoef, P. (2007). Marketing Communications Drivers of Adoption Timing of a New E-Service Among Existing Customers. Journal of Management, $71(2)$, 169-183.

Nowlis, S., Simonson, I. (1996). The Effect of New Product Features on Brand Choice. Journal of Marketing Research, 33(1), 36.

Thompson, S., Sinha, R. (2008). Brand Communities and New Product Adoption: The Influence ans Limits of Oppositional Loyalty. Journal of Marketing Management, $\quad 72(6), \quad 65-80$.

Resende, M., Zeidan, R. (2015). Psychological Biases and Economic Expectation: Evidence on Industry Experts. Journal of Neuroscience, Psychology, and Economics, $8(3)$

160-172.

Gordon, W. (2011). Behavioural Economics and Qualitative Research - A Marriage Made in Heaven? International Journal of Marketing Research, 53(2), 171-185.

Rick, A. L., Currim, I. (2005). An Experimental Investigation of Scanner Data Preparation Strategies for Consumer Choice Models. International Journal of Research in $\quad$ Marketing, $\quad 22(3), \quad 319-350$. 
Tammo, B. H., Van Heerde, H., Pieters, R. (2005). New Empirical Generalization on the Determinants of Price Elasticity. Journal of Marketing Research, 42(5), 141197.

Pradeep, K. C. (1993). Investigating Purchase Incidence, Brand Choice, and Purchase Quantity Decisions of Households. Marketing Science, 12(2), 184-208.

David, B. R., Chiang, J., Padmanadhan, V. (1999). The Descomposition of Promotional Response: An Empirical Generalization. Marketing Science., 18(4), 504-526.

Dmitri, K., Villas-Boas, M. (2008). Endogeneity and Individual Consumer Choice. $\begin{array}{llll}\text { Journal of } & \text { Marketing, } & \text { 75(12), }\end{array}$

Amil, P., Train, K. (2010). A Control Function Approach to Endogeneity in Consumer Choice Models. Journal of Marketing Research, 47(2), 3-13.

Backhaus, K., Hillig, T., Wilken, R. (2007). Predicting Purchase Decisions With Different Conjoint Analysis Methods. A Monte Carlo Simulation. International Journal of Marketing Research, 49(3), 341-364.

Dhar, R. (1997). Customer Prefference for Non-Choice Options. Journal of Customer Research, 24(2), 215-231.

SINNETIC. (2014). Construcción y validación de una escala para cuantificar cogniciones asociadas a los costos ocultos de elección de productos de consumo masivo en mercados colombianos y mexicanos. Bogotá: SINNETIC.

Santelli, A. G., Struthers, C. W., Eaton, J. (2009). Fit to Forgive: Exploring the interaction Between Regulatory Focus, Repentance, and Forgiveness.-. Journal of $\begin{array}{llll}\text { Personality and } & \text { Social } & \text { Psychology., } & \text { 96(2), }\end{array}$

McFall, J. P. (2015). Rational, Normative, Descriptive, Prescriptive, or Choice behavior? The Search for Integrative Metatheory of Decision Making. Behavioral $\begin{array}{lll}\text { Developments } & \text { Bulleting, } & \text { 20(1), }\end{array}$

Müller-Trede, J., Sher, S., McKenzie, C. R. (2015). Transitivity in Context: A Rational Analysis of Intransitive Choice and Context-Sensitive Preference. Decision, $2(4)$,

280-205 
White, C. M., Hofrage, U., Reisen, N. (2015). Choice Deferral Can Arrise From Absolte Evaluation or Relative Comparison. Journal of Experimental Psychology: Applied, $21(2)$ 140-157.

IBM. (2014). IBM(R) SPSS(R) Data Collection Interviewer 6.0.1. User's Guide. Chicago: IBM. Montero, I., Leon, O. G. (2007). A Guide for Naming Research in Psychology. International Journal of Clinical and Health Psychology, 7(3), 847-862.

SAS Institute. (2014). SAS Stat 9,3 Users Guide. Cary: SAS Press. Hensher, D. A., Rose, J. M., Greene, W. H. (2005). Applied Choice Analysis: A primer. Cambridge: Cambridge University

Press.

McFadden, D. (1974). Frontiers in Econometrics. En P. Zarembka, Conditional Logit Analysis of Cualitative Choice Behavior (págs. 105-150). New York: Academic Press.

Chorus, C. G. (2010). A New Random Regret Mininization . European Journal of Transport Infraestructure 181-277.

Bell, D. E. (1982). Regret in Decision Making Under Uncertainty. Operational Research, $30(5)$

961-1042.

Muller, H., Kroll, E. B., Vogt, B. (2012). Do Real Payments Really Matter? A Re-Examination of the Compromise Effect in Hypotetical and Binding Choice Set$\begin{array}{llll}\text { tings. } & \text { Marketing } & \text { Letters, } & \text { 23(1), }\end{array}$ Chorus., C. G. (2012). Random Regret-Based Discrete Choice Modelling: A Tutorial. Heidelberg: Springer

Simonson, I. (1992). The Influence of Anticipating Regret And Responsibility on Purchasing Decision. Journal of Consumer Research, 19(1), 105-124.

Kivetz, R., Netzer, O., Srinivasan, V. (2004). Models for Capturing the Compromise Effects. Journal of Marketing Research, 41, 237-294.

Hursh, S. R. (1980). Economic Concepts of Analysis of Behavior. Journal of Experimental Analysis of Behavior., 219-238.

Bekker-Grob, E., Ryan, M., Gerard, K. (2012). Discrete Choice Experiments In Health Economics: A Review of Literature. Health Economics, 21(2), 145-217. 
Mangham, L., Hanson, K., McPake, B. (2009). How to do (or not to do) ... Designing a discrete choice experiment for application in a low income Country. Health Policy Plan, $24(2)$

151-159.

Louviere, J., Hensher, D., Swait, J. (2000). Stated Choice Methods: Analysis and Application. . Cambridge: Cambridge University Press.

Hensher, D., Rose, J., Greene, W. (2005). Applied choice analysis: A Primer. Cambridge: Cambridge University Press;.

Louviere, J., Lancsar, E. (2009). Choice experiments in health: the good the bad, the ugly and toward a brighter future. Health Economy Policy, 4(4), 527-573.

McFadden, D. (1974). Conditional Logit Analysis of Qualitative Choice Behavior. En P. Zarembka, Frontiers in econometrics. (págs. 105-147). New York: Academic Press.

Arentze, T., Timmermans, H. (2007). Parametric action trees: incorporating continuous attribute variables into rule-based models of discrete choice. Transport Re$\begin{array}{llll}\text { search } & \text { Bulleting, } & \text { 41(7), }\end{array}$

Zhang, J., Timmermans, H., Borgers, A., Wang, D. (2004). Modelling traveler choice behavior using the concepts of relative utility and relevant interest. Transport Research Bulleting., $\quad 38(3), \quad$ 215-249.

Kivetz, R., Netzer, O., Srinivasan, V. (2004). Alternative models for capturing the compromise effects. Journald of Marketing Research., 41, 237-294.

Zeelenberg, M., Pieters, R. (2007). A theory of regret regulation 1.0. Journal of $\begin{array}{llll}\text { Consumer } & \text { Psychology, } & \text { 3-21. }\end{array}$

DANE. (2011). Resultados de la encuesta nacional agropecuaria ENA. Bogotá Colombia.: Dirección de metodología y producción estadística DIMPE.

Velandia, A., López, W. (2008). Investigación cualitativa y psicología del consumidor: alternativas de aplicación. Avances en Psicología Latinoamericana., 26(2), 290-303.

Franzosi, R., Doyle, S., McCelland, L., Putnam Rankin, C., Vicari, S. (2013). Quantitative narrative analysis software option compared: PC-ACE and CAQDAS (ATLAS.ti, MAXqda, and Nvivo). Quality and Quantity, 47(6), 3219-3247. 
Anderson, D., Wiley, J. (1992). Efficient choice ser designs for estimating availability cross.effects models. Marketing Letters., 2(4), 357-370.

Corrigan, J., Depositario, D., Nayga Jr, R., Wu, X., Laude, T. (2009). Comparing open-ended choice experiments and experimental auctions: An application to golden rice. American Journal of Agricultural Economics., 91(3), 837-853.

Howard-Payne, L. (2016). Glaser or Strauss? Considerations for selecting a grounded theory study. South African Journal of Psychology, 46(1), 50-62.

Sussex, J., Towse, A., Devlin, N. (2013.). Operationalizing Value-Based Pricing of Medicines: A Taxonomy of Approaches. PharmacoEconomics., 3, 1-10.

Karimi, A., Siarry, P. (2012). Global Simplex Optimization-A simple and efficient metaheuristic for continuous optimization. Engineering Applications of Artificial Intelligence., $\quad 25(1), \quad 48-55$.

Richardson, J., McKie, J. (2005). Empiricism, ethics and orthodox economic theory: what is the appropriate basis for decision-making in the health sector? Social Science $\quad$ Medicine, 60(2), 265-275.

Kahneman, D. (2011). Thinking, fast and slow. New York: Farrar, Straus and Giroux.

Cranenburgh, S., Angelo Guevara, C., Chorus, C. (2015). New insights on random regret minimization models. Transportation Research., 74, 91-109.

Chorus, C. (2012). Random regret minimization: an overview of model properties and empirical evidence. Transport Reviews, 32(1), 75-92.

McGilchrist, , C. (1994). Estimation in generalized mixed models. Journal of the Royal Statistical Society: Series B (Methodological), 56(1), 61-69.

Heydari , S., Miranda-Moreno, L., Liping, , F. (2014). Speed limit reduction in urban areas: A before-after study using Bayesian generalized mixed linear models. Accident Analysis $\quad$ Prevention, $\quad 73, \quad 252-261$. 